\title{
Differential Equations with Tempered $\Psi$-Caputo Fractional Derivative
}

\author{
Milan Medved ${ }^{a}$ and Eva Brestovanská ${ }^{b}$ \\ ${ }^{a}$ Department of Mathematical Analysis and Numerical Mathematics, Faculty \\ of Mathematics, Physics and Informatics, Comenius University \\ Mlynská dolina, 84248 Bratislava, Slovakia \\ ${ }^{b}$ Department of Economics and Finance, Faculty of Management, Comenius \\ University \\ Odbojárov 10, 83104 Bratislava, Slovakia \\ E-mail(corresp.): milan.medved@fmph.uniba.sk \\ E-mail: eva.brestovanska@fm.uniba.sk
}

Received August 4, 2020; revised September 29, 2021; accepted September 29, 2021

\begin{abstract}
In this paper we define a new type of the fractional derivative, which we call tempered $\Psi$-Caputo fractional derivative. It is a generalization of the tempered Caputo fractional derivative and of the $\Psi$-Caputo fractional derivative. The Cauchy problem for fractional differential equations with this type of derivative is discussed and some existence and uniqueness results are proved. We present a Henry-Gronwall type inequality for an integral inequality with the tempered $\Psi$-fractional integral. This inequality is applied in the proof of an existence theorem. A result on a representation of solutions of linear systems of $\Psi$-Caputo fractional differential equations is proved and in the last section an example is presented.
\end{abstract}

Keywords: tempered Riemann-Liouville fractional derivative, tempered $\Psi$-Caputo fractional derivative.

AMS Subject Classification: 34A08; 34A12; 34A40; 34D05; 34A34.

\section{Introduction}

Fractional calculus, introduced by L. Liouville [24] and B. Riemann [36], is a powerful tool for the study of systems describing various real memory processes. The classical Riemann-Liouville fractional derivative is very often used in applications in physics, chemistry, biology and also in other sciences such as economics, geology, sociology. Several other types of fractional derivatives

Copyright (C) 2021 The Author(s). Published by Vilnius Gediminas Technical University

This is an Open Access article distributed under the terms of the Creative Commons Attribution License (http://creativecommons.org/licenses/by/4.0/), which permits unrestricted use, distribution, and reproduction in any medium, provided the original author and source are credited. 
have also been defined in recent decades. They are more general and more suitable for applications than the Riemann-Liouville fractional derivative. Some definitions of such fractional derivatives can be found in the remarkable monographs $[13,16,19,22,33,35,45,48]$ on fractional calculus and qualitative analysis of fractional-order differential equations. In the recently published papers $[12,23,37]$ so-called tempered Riemann-Liouville and tempered Caputo fractional derivatives are defined. Fractional differential equations with these derivatives have very good properties from the point of view of stability of their solutions. It is well-known that the classical fractional differential equations can have asymptotically stable solutions, but not exponentially. Some sufficient conditions for the exponential stability of solutions of fractionally perturbed ODEs with tempered Riemann-Liouville fractional integrals and several constant delays are proved in the papers $[28,29,30,31]$. A new type of derivative, called the Hilfer fractional derivative, was defined by R. Hilfer [16]. In the paper [39] a new definition of fractional derivative, called $\Psi$-Hilfer fractional derivative, is defined.

Recently, many papers are devoted to fractional differential equations with $\Psi$-Hilfer fractional derivative. Some existence results are obtained in the papers $[1,5,8,10,14,21]$ and stability results in $[2,3,4,6,7,20,25,40,43,44,46]$. Properties of $\Psi$-Hilfer fractional derivative and $\Psi$-Hilfer integral are studied in the papers [42] and [39]. In the papers [9,41], the Gronwall inequality is generalized to some classes of integral inequalities with $\Psi$-Hilfer integral.

Inspired by the papers $[10,39,41]$, we define a modification of the $\Psi$-Caputo derivative, which we call the tempered $\Psi$-Caputo derivative and we study the Cauchy problem for fractional differential equations with this type of fractional derivative. This derivative includes as special cases the tempered Caputo [23].

The main aim of this paper is to present some results on the existence and uniqueness of solutions of fractional differential equations with the tempered $\Psi$-Caputo derivative.

\section{Preliminaries}

In this section, we recall definitions of known fractional derivatives (see e.g. $[12,23,32])$.

Definition 1. Suppose that real function $u(t)$ is piecewise continuous, integrable on $(a, b), \alpha>0, \lambda \geq 0$. The tempered Riemann-Liouville fractional integral of order $\alpha$ is defined to be

$$
I_{a}^{\alpha, \lambda} u(t)=e^{-\lambda t} I_{a}^{\alpha}\left(e^{\lambda t} u(t)\right)=\frac{1}{\Gamma(\alpha)} \int_{a}^{t}(t-s)^{\alpha-1} e^{-\lambda(t-s)} u(s) d s
$$

where $I_{a}^{\alpha} u(t)$ denotes the Riemann-Liouville fractional integral

$$
I_{a}^{\alpha} u(t)=\frac{1}{\Gamma(\alpha)} \int_{a}^{t}(t-s)^{\alpha-1} u(s) d s
$$


Definition 2. For $n-1<\alpha<n, n \in \mathbb{N}, \lambda \geq 0$ the tempered RiemannLiouville fractional derivative of order $\alpha$ is defined to be

$$
D_{a}^{\alpha, \lambda} u(t)=e^{-\lambda t} D_{a}^{\alpha}\left(e^{\lambda t} u(t)\right)=\frac{e^{-\lambda t}}{\Gamma(n-\alpha)} \frac{d^{n}}{d t^{n}} \int_{a}^{t}(t-s)^{n-\alpha-1} e^{\lambda s} u(s) d s,
$$

where $D_{a}^{\alpha}\left(e^{\lambda t} u(t)\right)$ denotes the Riemann-Liouville fractional derivative

$$
D_{a}^{\alpha}\left(e^{\lambda t} u(t)\right)=\frac{d^{n}}{d t^{n}}\left(I_{a}^{n-\alpha}\left(e^{\lambda t} u(t)\right)\right)=\frac{1}{\Gamma(n-\alpha)} \frac{d^{n}}{d t^{n}} \int_{a}^{t}(t-s)^{n-\alpha-1} e^{\lambda s} u(s) d s
$$

for $u \in C[a, b]$.

Definition 3. For $n-1<\alpha<n, n \in \mathbb{N}, \lambda \geq 0$ the tempered Caputo, or $\lambda$-tempered fractional derivative of order $\alpha$ is defined to be

$$
{ }^{C} D_{a}^{\alpha, \lambda} u(t)=e^{-\lambda t C} D_{a}^{\alpha}\left(e^{\lambda t} u(t)\right)=\frac{e^{-\lambda t}}{\Gamma(n-\alpha)} \int_{a}^{t}(t-s)^{n-\alpha-1} \frac{d^{n}}{d s^{n}}\left(e^{\lambda s} u(s)\right) d s,
$$

where ${ }^{C} D_{a}^{\alpha}\left(e^{\lambda t} u(t)\right)$ denotes the Caputo fractional derivative

$$
{ }^{C} D_{a}^{\alpha}\left(e^{\lambda t} u(t)\right)=\frac{1}{\Gamma(n-\alpha)} \int_{a}^{t}(t-s)^{n-\alpha-1} \frac{d^{n}}{d s^{n}}\left(e^{\lambda s} u(s)\right) d s
$$

for $u \in C^{n}[a, b]$.

Obviously, ${ }^{C} D_{a}^{\alpha, 0} u(t)={ }^{C} D_{a}^{\alpha} u(t)$. This type of derivative was first defined in the article [37] (see also [12,23,32]).

Definition 4 . Let $\alpha>0$, the real function $u(t)$ be continuous on $[a, b]$ and $\Psi \in C^{1}[a, b]$ is an increasing differentiable function such that $\Psi^{\prime}(t) \neq 0$ for all $t \in[a, b]$. Then the $\Psi$-Riemann-Liouville fractional integral of order $\alpha$ is defined by

$$
I_{a}^{\alpha, \Psi} u(t)=\frac{1}{\Gamma(\alpha)} \int_{a}^{t}[\Psi(t)-\Psi(s)]^{\alpha-1} \Psi^{\prime}(s) u(s) d s
$$

(see $[39,41])$.

Now, we define a new type of $\Psi$-fractional integral, so-called tempered $\Psi$-fractional integral.

Definition 5. Let the assumptions of the Definition 4 be satisfied and let $\lambda \geq 0$. Then the tempered $\Psi$-fractional integral of order $\alpha$ is defined by

$$
\begin{aligned}
I_{a}^{\alpha, \lambda, \Psi} u(t) & \left.=e^{-\lambda \Psi(t)} I^{\alpha, \Psi}\left(e^{\lambda \Psi(t)} u(t)\right)\right) \\
& =\frac{1}{\Gamma(\alpha)} \int_{a}^{t}[\Psi(t)-\Psi(s)]^{\alpha-1} e^{-\lambda[\Psi(t)-\Psi(s)]} \Psi^{\prime}(s) u(s) d s
\end{aligned}
$$




\section{Tempered $\Psi$-Caputo fractional derivative}

In this section, we introduce a new type of fractional derivative, which includes the above defined $\Psi$-Caputo fractional derivative, by the following definition.

Definition 6. Let $\Psi \in C^{n}[a, b]$ is an increasing differentiable function such that $\Psi^{\prime}(t) \neq 0$ for all $t \in[a, b]$. For $n-1<\alpha<n, n \in \mathbb{N}, \lambda \geq 0$ the tempered $\Psi$-Caputo fractional derivative of order $\alpha$ is defined to be

$$
{ }^{C} D_{a}^{\alpha, \lambda, \Psi} x(t)=\frac{e^{-\lambda \Psi(t)}}{\Gamma(n-\alpha)} \int_{a}^{t} \Psi^{\prime}(s)(\Psi(t)-\Psi(s))^{n-\alpha-1} x_{\lambda, \Psi}^{[n]}(s) d s,
$$

where

$$
x_{\lambda, \Psi}^{[n]}(t)=\left[\frac{1}{\Psi^{\prime}(t)} \frac{d}{d t}\right]^{n}\left(e^{\lambda \Psi(t)} x(t)\right) .
$$

The $\Psi$-Caputo fractional derivative, introduced in the paper [10], is defined by the rule

$$
{ }^{C} D_{a}^{\alpha, \Psi} x(t)=\frac{1}{\Gamma(n-\alpha)} \int_{a}^{t} \Psi^{\prime}(s)[\Psi(t)-\Psi(s)]^{n-\alpha-1} x_{\Psi}^{[n]} x(s) d s,
$$

where

$$
x_{\Psi}^{[n]} x(t)=\left[\frac{1}{\Psi^{\prime}(t)} \frac{d}{d t}\right]^{n}(x(t)) .
$$

It is clear that ${ }^{C} D_{a}^{\alpha, 0, \Psi} x(t)={ }^{C} D_{a}^{\alpha, \Psi} x(t)$ and $x_{0, \alpha, \Psi}^{[n]}(t)=x_{\Psi}^{[n]} x(t)$. Obviously,

$$
{ }^{C} D_{a}^{\alpha, \lambda, \Psi} x(t)=e^{-\lambda \Psi(t) C} D_{a}^{\alpha, \Psi}\left(e^{\lambda \Psi(t)} x(t)\right) .
$$

The definition of the tempered $\Psi$-Caputo fractional derivative covers the wellknown fractional derivatives for $\lambda=0$, like the Caputo fractional derivative $(\Psi(t)=t)$, the Caputo-Hadamard fractional derivative $(\Psi(t)=\ln t)$, the Caputo-Erdélyi-Kober fractional derivative $\left(\Psi(t)=t^{\sigma}\right)$. The tempered $\Psi$-Caputo derivative with $\Psi(t)=t$, is defined and considered in the papers $[12,23]$. We need the following lemma, proved in [10, Theorem 1].

Lemma 1. Let $n-1<\alpha<n$ and $h:[a, b] \rightarrow \mathbb{R}$. Then the following holds:

1. If $h \in C[a, b]$, then ${ }^{C} D_{a}^{\alpha, \Psi} I_{a}^{\alpha, \Psi} h(t)=h(t)$.

2. If $h \in C^{n}[a, b]$, then $I_{a}^{\alpha, \Psi C} D_{a}^{\alpha, \Psi} h(t)=h(t)-\sum_{k=0}^{n-1} c_{k}[\Psi(t)-\Psi(a)]^{k}$, where $c_{k}=h_{\Psi}^{[k]}(a) / k !$.

Theorem 1. (Composition properties). Let the assumptions of Definition 5 and Definition 6 be satisfied. Then the following holds:

$$
\begin{aligned}
& \text { 1. }{ }^{C} D_{a}^{\alpha, \lambda, \Psi}\left[I_{a}^{\alpha, \lambda, \Psi} x(t)\right]=x(t), \\
& \text { 2. } I_{a}^{\alpha, \lambda, \Psi}\left[{ }^{C} D_{a}^{\alpha, \lambda, \Psi} x(t)\right]=x(t)-e^{-\lambda \Psi(t)} \sum_{k=0}^{n-1} c_{k}[\Psi(t)-\Psi(a)]^{k},
\end{aligned}
$$


where

$$
c_{k}=\frac{x_{\lambda, \Psi}^{[k]}(a)}{k !}=\left.\frac{1}{k !}\left[\frac{1}{\Psi^{\prime}(t)} \frac{d}{d t}\right]^{k}\left(e^{\lambda \Psi(t)} x(t)\right)\right|_{t=a}
$$

Proof. Let us prove the assertion 1.

$$
\begin{aligned}
{ }^{C} D_{a}^{\alpha, \lambda, \Psi}\left[I_{a}^{\alpha, \lambda, \Psi} x(t)\right] & =e^{-\lambda \Psi(t) C} D_{a}^{\alpha, \Psi}\left(e^{\lambda \Psi(t)}\left(I_{a}^{\alpha, \Psi} x(t)\right)\right) \\
& =e^{-\lambda \Psi(t) C} D_{a}^{\alpha, \Psi}\left(e^{-\lambda \Psi(t)} e^{\lambda \Psi(t)}\left(I_{a}^{\alpha, \Psi}\left(e^{\lambda \Psi(t)} x(t)\right)\right)\right) \\
& =e^{-\lambda \Psi(t) C} D_{a}^{\alpha, \Psi} I_{a}^{\alpha, \Psi}\left(e^{\lambda \Psi(t)} x(t)\right)=x(t) .
\end{aligned}
$$

Next, we prove the assertion 2 .

$$
\begin{aligned}
I_{a}^{\alpha, \lambda, \Psi}\left[{ }^{C} D_{a}^{\alpha, \lambda, \Psi} x(t)\right] & =e^{-\lambda \Psi(t)} I_{a}^{\alpha, \Psi}\left(e^{\lambda \Psi(t)}\left[e^{-\lambda \Psi(t) C} D_{a}^{\alpha, \Psi}\left(e^{\lambda \Psi(t)} x(t)\right)\right]\right) \\
& =e^{-\lambda \Psi(t)} I_{a}^{\alpha, \Psi C} D_{a}^{\alpha, \Psi}\left(e^{\lambda \Psi(t)} x(t)\right) .
\end{aligned}
$$

From the assertion 1 of Lemma 1, we have

$$
I_{a}^{\alpha, \lambda, \Psi C} D_{a}^{\alpha, \lambda, \Psi} x(t)=e^{-\lambda \Psi(t)}\left[e^{\lambda \Psi(t)} x(t)\right]-e^{-\lambda \Psi(t)} \sum_{k=0}^{n-1} c_{k}[\Psi(t)-\Psi(a)],
$$

where $c_{k}=\frac{x_{\lambda, \Psi}^{[k]}(a)}{k !}=\left.\frac{1}{k !}\left[\frac{1}{\Psi^{\prime}(t)} \frac{d}{d t}\right]^{k}\left(e^{\lambda \Psi(t)} x(t)\right)\right|_{t=a}$.

\section{Proposition 1.}

$$
\lim _{\alpha \rightarrow(n-1)^{+}}{ }^{C} D_{a}^{\alpha, \lambda, \Psi} x(t)=e^{-\lambda \Psi(t)}\left[x_{\lambda, \Psi}^{[n-1]}(t)-x_{\lambda, \Psi}^{[n-1]}(a)\right] .
$$

Proof. From the definition of tempered $\Psi$-Caputo derivative we obtain

$$
\begin{aligned}
& \lim _{\alpha \rightarrow(n-1)^{+}}{ }^{C} D_{a}^{\alpha, \lambda, \Psi} x(t)=e^{-\lambda \Psi(t)} \int_{a}^{t} \Psi^{\prime}(s) x_{\lambda, \Psi}^{[n]}(s) d s \\
& =e^{-\lambda \Psi(t)} \int_{a}^{t} \Psi^{\prime}(s)\left(\frac{1}{\Psi^{\prime}(s)} \frac{d}{d s} x_{\lambda, \Psi}^{[n-1]}(s)\right) d s=e^{-\lambda \Psi(t)} \int_{a}^{t} \frac{d}{d s} x_{\lambda, \Psi}^{[n-1]}(s) d s \\
& =e^{-\lambda \Psi(t)}\left[x_{\lambda, \Psi}^{[n-1]}(t)-x_{\lambda, \Psi}^{[n-1]}(a)\right] .
\end{aligned}
$$

\section{Henry-Gronwall type integral inequality with tempered $\Psi$-fractional integral}

The first fractional version of the well-known Gronwall inequality can be found in the book by D. Henry [15, Lemma 7.1.1], devoted to the study of qualitative properties of semilinear parabolic equations. In the paper [47], a generalization of the Henry-Gronwall inequality is proved. This result is recently frequently applied in the study of the existence and uniqueness of solutions of Cauchy 
initial value problems for fractional differential equations. In the paper [9], a generalization of the Gronwall inequality for integral inequality with the Katugampola fractional integral (see [18]) is presented. The first nonlinear versions of the Henry-Gronwall inequality were proved in the papers [26,27], where a desingularization method was developed. A Henry-Gronwall type of integral inequality with the $\Psi$-fractional integral is presented in the paper [41] and it is used there in the proof of a result on the continuous dependence of solutions of the Cauchy initial value fractional problem with the $\Psi$-Hilfer fractional derivative.

Next, we present a Henry-Gronwall type inequality for an integral inequality with the tempered $\Psi$-fractional integral. We will apply this inequality in the next section to prove a uniqueness result. The key role in the proof plays the following lemma that is a consequence of a nonlinear version of the Bihari inequality, published in [11, Theorem].

Lemma 2. Let $0<T<\infty, c<T, a(t), b(t), k(t), v(t)$ be nonnegative, continuous functions on the interval $[c, T]$ and let

$$
v(t) \leq a(t)+b(t) \int_{c}^{t} k(s) v(s) d s, \forall c \leq t \leq T
$$

Then

$$
v(t) \leq A(t) e^{B(t) \int_{c}^{t} k(s) d s}, \forall c \leq t \leq T
$$

where $A(t)=\sup _{c \leq s \leq t} a(s), B(t)=\sup _{c \leq s \leq t} b(s)$.

Theorem 2. Let $\lambda \geq 0, \alpha \in(0,1), p>1, p(\alpha-1)+1>0, q=\frac{p}{p-1}$, $a(t), b(t), F(t)$ be nonnegative, integrable functions on the interval $[0, T)$, where $0<T<\infty, \Psi$ is a continuously differentiable, increasing function on the interval $[0, \infty)$ with $\Psi(0)=0, \Psi^{\prime}(t)>0$ for all $t \in(0, \infty), \lim _{t \rightarrow \infty} \Psi(t)=\infty$ and $u(t)$ be a nonnegative integrable function on $[0, T)$, satisfying the inequality

$$
u(t) \leq a(t)+b(t) \int_{0}^{t}[\Psi(t)-\Psi(s)]^{\alpha-1} e^{-\lambda[\Psi(t)-\Psi(s)]} \Psi^{\prime}(s) F(s) u(s) d s, 0 \leq t \leq T .
$$

Then

$$
u(t) \leq C(t) e^{D(t) \int_{0}^{t} \Psi^{\prime}(s) F(s)^{q} d s}, 0 \leq t \leq T
$$

where

$$
C(t)=2^{\frac{q-1}{q}} \sup _{0 \leq s \leq t} a(s), D(t)=\frac{2^{q-1}}{q} M_{p}^{q} \sup _{0 \leq s \leq t} b(s)^{q}, M_{p}=\left(\frac{\Gamma(p(\alpha-1)+1}{(p \lambda)^{p(\alpha-1)+1}}\right)^{\frac{1}{p}} .
$$

Proof. Let us estimate the integral from the right-hand side of the Inequality (4.1). Since $1 / p+1 / q=1$, we can write $\Psi^{\prime}(t)=\Psi^{\prime}(t)^{1 / p} \Psi^{\prime}(t)^{1 / q}$ and by 
using the Hölder inequality we obtain

$$
\begin{aligned}
& \int_{0}^{t}[\Psi(t)-\Psi(s)]^{\alpha-1} e^{-\lambda[\Psi(t)-\Psi(s)]} \Psi^{\prime}(s) F(s) u(s) d s \\
& =\int_{0}^{t}\left([\Psi(t)-\Psi(s)]^{\alpha-1} e^{-\lambda[\Psi(t)-\Psi(s)]}\left[\Psi^{\prime}(s)\right]^{1 / p}\right)\left(\left[\Psi^{\prime}(s)\right]^{1 / q} F(s) u(s)\right) d s \\
& \leq\left(\int_{0}^{t}[\Psi(t)-\Psi(s)]^{p(\alpha-1)} e^{-p \lambda[\Psi(t)-\Psi(s)]} \Psi^{\prime}(s) d s\right)^{\frac{1}{p}}\left(\int_{0}^{t} \Psi^{\prime}(s) F(s)^{q} u(s)^{q} d s\right)^{\frac{1}{q}} \\
& =\left(\int_{0}^{\Psi(t)} \sigma^{p(\alpha-1)} e^{-p \lambda \sigma} d \sigma\right)^{1 / p}\left(\int_{0}^{t} \Psi^{\prime}(s) F(s)^{q} u(s)^{q} d s\right)^{1 / q} .
\end{aligned}
$$

One can show that

$$
\left(\int_{0}^{\Psi(t)} \sigma^{p(\alpha-1)} e^{-p \lambda \sigma} d \sigma\right)^{1 / p} \leq\left(\int_{0}^{\infty} \sigma^{p(\alpha-1)} e^{-p \lambda \sigma} d \sigma\right)^{1 / p}=M_{p}
$$

where $M_{p}=\left(\Gamma(p(\alpha-1)+1) /(p \lambda)^{p(\alpha-1)+1}\right)^{1 / p}$. Hence, from the Inequalities (4.1) and (4.2) we obtain the inequality

$$
u(t) \leq a(t)+M_{p} b(t)\left(\int_{0}^{t} \Psi^{\prime}(s) F(s)^{q} u(s)^{q} d s\right)^{1 / q} .
$$

Using the inequality $(c+d)^{q} \leq 2^{q-1}\left(c^{q}+d^{q}\right)$, valid for any nonnegative numbers $c, d$, we obtain

$$
u(t)^{q} \leq \tilde{a}(t)+\tilde{b}(t) \int_{0}^{t} \Psi^{\prime}(s) F(s)^{q} u(s)^{q} d s,
$$

where $\tilde{a}(t)=2^{q-1} a(t)^{q}, \tilde{b}(t)=2^{q-1} M_{p}^{q} b(t)^{q}$. From the Inequality (4.3) and Lemma 2 it follows

$$
u(t)^{q} \leq A(t) e^{B(t) \int_{0}^{t} \Psi^{\prime}(s) F(s)^{q} d s} t \in[0, T),
$$

where

$$
A(t)=2^{q-1} \sup _{0 \leq s \leq t} a(s)^{q}, B(t)=2^{q-1} M_{p}^{q} \sup _{0 \leq s \leq t} b(s)^{q},
$$

and the Inequality (4.4) yields

$$
u(t) \leq C(t) e^{D(t) \int_{0}^{t} \Psi^{\prime}(s) F(s)^{q} d s},
$$

where

$$
C(t)=2^{\frac{q-1}{q}} \sup _{0 \leq s \leq t} a(s), D(t)=\frac{2^{q-1}}{q} M_{p}^{q} \sup _{0 \leq s \leq t} b(s)^{q} .
$$

Obviously, the following consequence of this theorem is valid. 
Corollary 1. Let $a(t) \equiv a, b(t) \equiv b$ be nonnegative constants, $F(t), \Psi(t)$ be as in the Theorem 2 and let $u(t)$ be a nonnegative integrable function satisfying the integral inequality

$$
u(t) \leq a+b \int_{0}^{t}[\Psi(t)-\Psi(s)]^{\alpha-1} e^{-\lambda[\Psi(t)-\Psi(s)]} \Psi^{\prime}(s) F(s) u(s) d s, \forall t \in[0, \infty) .
$$

Then

$$
u(t) \leq c e^{d \int_{0}^{t} \Psi^{\prime}(s) F(s)^{q} d s} \forall t \in[0, \infty),
$$

where $c=2^{\frac{q-1}{q}} a, d=2^{\frac{q-1}{q}} M_{p}^{q} b^{q}$.

Remark 1 . The numbers $a, b, M_{p}$ in the Inequality (4.5) are independent of $T$ and therefore the Corollary 1 can be formulated for $T=\infty$.

Example 1. If $\psi(t) \equiv t$, then the Inequality (4.6) has the form

$$
u(t) \leq c e^{d \int_{0}^{t} F(s)^{q} d s} \forall t \in[0, T) .
$$

Example 2. If $\psi(t) \equiv \ln (1+t), F(t) \equiv 1$, then the Inequality (4.6) has the form

$$
u(t) \leq c(1+t)^{d} \forall t \in[0, T) .
$$

Proof.

$$
u(t) \leq c e^{d \Psi(t)}=c e^{d \ln (1+t)}=c e^{\ln (1+t)^{d}}=c(1+t)^{d} \forall 0 \leq t<T .
$$

Example 3. If $\alpha=1, \lambda=0$ and $\psi(t) \equiv \ln (1+t), F(t) \equiv 1$, then the Inequality (4.5) has the form

$$
u(t) \leq a+b \int_{0}^{t} \frac{1}{1+s} u(s) d s \forall t \in[0, T)
$$

and the classical Gronwall lemma yields the inequality

$$
u(t) \leq a(1+t)^{b} \forall t \in[0, T) .
$$

This means that the result from the Example 2 is a fractional version of the this result.

\section{Fractional differential equations with tempered $\Psi$-Caputo derivative}

In this section, we consider the following initial value problem

$$
\begin{aligned}
{ }^{C} D_{0}^{\alpha, \lambda, \Psi} x(t) & =f(t, x(t)), \quad t \in[0, a], \alpha \in(0,1), \\
x(0) & =x_{0} \in \mathbb{R}^{n},
\end{aligned}
$$

where $f \in C\left([0, a] \times \mathbb{R}^{n}, \mathbb{R}^{n}\right), \Psi$ is a continuously differentiable, increasing function on the interval $[0, \infty)$ with $\Psi(0)=0, \Psi^{\prime}(t)>0$ for all $t \in(0, \infty), \lim _{t \rightarrow \infty} \Psi(t)$ $=\infty$. For simplicity in notation, we write $C_{a}$ instead of $C\left([0, a], \mathbb{R}^{n}\right)$. We shall denote by $|\cdot|$ the norm on $\mathbb{R}^{n}$ and $\|g\|_{C_{a}}=\sup _{t \in[0, a]} g(t)$ for $g \in C_{a}^{0}$. 
Definition 7. A mapping $x:[0, a] \rightarrow \mathbb{R}^{n}$ is a solution of the initial value problem (5.1)- (5.2), if it is continuous, ${ }^{C} D_{0}^{\alpha, \lambda, \Psi} x(t)$ exists for all $t \in(0, a]$ and is continuous on $(0, a], x(t)$ fulfills the Equality (5.1) for all $t \in(0, a]$ and the initial condition (5.2).

Lemma 3. Let the mapping $f \in C\left([0, a] \times R^{n}, R^{n}\right)$ be bounded. Then the mapping $x(t)$ is a solution of the initial value problem (5.1)-(5.2) defined on the interval $[0, a]$ if and only if it is a solution of the equation

$$
\begin{aligned}
x(t)= & e^{-\lambda \Psi(t)} x_{0} \\
& +\frac{1}{\Gamma(\alpha)} \int_{0}^{t}[\Psi(t)-\Psi(s)]^{\alpha-1} e^{-\lambda[\Psi(t)-\Psi(s)]} \Psi^{\prime}(s) f(s, x(s)) d s,
\end{aligned}
$$

for all $t \in[0, a]$.

Proof. Necessity. Performing the integral $I_{0}^{\lambda, \alpha, \Psi}$ to both sides of the Equation (5.1) and applying the assertion 2 of Theorem 1, we obtain the Equation (5.3). Sufficiency. Since ${ }^{C} D_{0}^{\alpha, \Psi}\left(x_{0}\right)=0$, we have

$$
{ }^{C} D_{0}^{\alpha, \lambda, \Psi}\left(e^{-\lambda \Psi(t)} x_{0}\right)=e^{-\lambda \Psi(t) C} D_{0}^{\alpha, \Psi}\left(e^{\lambda \Psi(t)} e^{-\lambda \Psi(t)}\left(x_{0}\right)\right)=0 .
$$

Applying the operator ${ }^{C} D_{0}^{\lambda, \alpha, \Psi}$ to both sides of the Equation (5.3), using equality (5.4) and the assertion 1 of Theorem 1, we obtain that $x(t)$ is a solution of the Equation (5.1). Putting $t=0$ in the formula (5.3) we obtain that $x(0)=x_{0}$ and the proof is finished.

Theorem 3. Let $\Psi \in C^{1}[0, \infty)$ be a nonnegative and increasing function such that $\Psi^{\prime}(t) \neq 0$ for all $t \in[0, \infty), \lim _{t \rightarrow \infty} \Psi(t)=\infty$ and $D=[0, \infty) \times \mathbb{R}^{n}$. Assume that the mapping $f: D \rightarrow \mathbb{R}^{n}$ is continuous bounded and satisfying the Lipschitz condition

$$
|f(t, x)-f(t, y)| \leq L|x-y| \quad \text { for all } \quad(t, x),(t, y) \in D,
$$

where $L>0$ is a constant, $\frac{L}{\lambda^{\alpha}}<1$ and $|$.$| is a norm on \mathbb{R}^{n}$. Then there is a unique solution $x(t)$ of the initial value problem (5.1)-(5.2) on the whole interval $[0, \infty)$.

Proof. Let $a$ be an arbitrary positive number. Define the set $G_{\gamma}=\{y \in$ $\left.C\left([0, a], \mathbb{R}^{n}\right): \max _{t \in[0, a]}\left|y(t)-x_{0} e^{-\lambda \Psi(t)}\right| \leq \gamma\right\}$, where

$$
\gamma=\frac{M}{\lambda^{\alpha}}, M=\sup _{(t, x) \in D}|f(t, x)| .
$$

The set $G_{\gamma}$ is a closed subset of the Banach space $(C[0, a],\|\cdot\|)$, where $\|g\|=$ $\max _{t \in[0, a]}|g(t)|$ and $|$.$| is a norm in \mathbb{R}^{n}$.

Let us define the operator $T: G_{\gamma} \rightarrow C\left([0, a], \mathbb{R}^{n}\right)$ by

$$
\begin{aligned}
(T x)(t)= & e^{-\lambda \Psi(t)} x_{0} \\
& +\frac{1}{\Gamma(\alpha)} \int_{0}^{t}[\Psi(t)-\Psi(s)]^{\alpha-1} e^{-\lambda[\Psi(t)-\Psi(s)]} \Psi^{\prime}(s) f(s, x(s)) d s .
\end{aligned}
$$


First, we prove that $T\left(G_{\gamma}\right) \subset G_{\gamma}$. Let $x \in G_{\gamma}, t \in[0, a]$. Since $|f(t, u)| \leq M$ for all $(t, u) \in D$, we have

$$
\begin{aligned}
& \left|(T x)(t)-x_{0} e^{-\lambda \Psi(t)}\right| \leq \frac{1}{\Gamma(\alpha)} \int_{0}^{t}[\Psi(t)-\Psi(s)]^{\alpha-1} e^{-\lambda[\Psi(t)-\Psi(s)]} \Psi^{\prime}(s)|f(s, x(s))| d s \\
& \leq \frac{M}{\Gamma(\alpha)} \int_{0}^{t}[\Psi(t)-\Psi(s)]^{\alpha-1} e^{-\lambda[\Psi(t)-\Psi(s)]} \Psi^{\prime}(s) d s=\frac{M}{\Gamma(\alpha)} \int_{0}^{\Psi(t)} \sigma^{\alpha-1} e^{-\lambda \sigma} d \sigma \\
& \leq \frac{M}{\Gamma(\alpha)} \int_{0}^{\infty} \sigma^{\alpha-1} e^{-\lambda \sigma} d \sigma=\frac{M}{\lambda^{\alpha}}=\gamma .
\end{aligned}
$$

Hence, $T\left(D_{\gamma}\right) \subset D_{\gamma}$. Now, we prove that $T: D_{\gamma} \rightarrow D_{\gamma}$ is a contraction. For $y, z \in D_{\gamma}, t \in[0, a]$, we have

$$
\begin{aligned}
& \|T(y)-T(z)\|_{C_{a}}=\max _{t \in[0, a]}|T(y)(t)-T(z)(t)| \\
& \leq \frac{L}{\Gamma(\alpha)} \max _{t \in[0, a]}\left(\int_{0}^{t}[\Psi(t)-\Psi(s)]^{\alpha-1} e^{-\lambda[\Psi(t)-\Psi(s)]} \Psi^{\prime}(s) d s\right)\|y-z\|_{C_{a}} \\
& \leq \frac{L}{\Gamma(\alpha)} \frac{\Gamma(\alpha)}{\lambda^{\alpha}}\|y-z\|_{C_{a}} \leq \frac{L}{\lambda^{\alpha}}\|y-z\|_{C_{a}} .
\end{aligned}
$$

Since by the assumption $\frac{L}{\lambda^{\alpha}}<1$, the operator $T: D_{\gamma} \rightarrow D_{\gamma}$ is a contraction. Hence by the Banach fixed point theorem the mapping $T$ has a unique fixed point. This means there is a unique solution $x(t)$ of the initial value problem (5.1)-(5.2) on any compact interval $[0, a]$. Since the contraction constant $\frac{L}{\lambda^{\alpha}}$ for the operator $T$ is independent of $a$, we have proven that there is a unique solution of this problem on the whole interval $[0, \infty)$.

In the proof of the next existence theorem, we will apply the following Schaefer's Fixed Point Theorem [38].

Theorem 4. Let $X$ be a Banach space and $T: X \rightarrow X$ be a completely continuous operator. If the set

$$
X(T)=\{y \in X: y=\mu T y \text { for some } \mu \in(0,1)\}
$$

is bounded, then $T$ has at least one fixed point.

Theorem 5. Let $\Psi \in C^{1}[0, a]$ be a nonnegative, increasing function with $\Psi^{\prime}(t)>0$ for all $t \in[0, a], \lim _{t \rightarrow \infty} \Psi(t)=\infty$ and $D=[0, a] \times \mathbb{R}^{n}, f: D \rightarrow \mathbb{R}^{n}$ is a continuous mapping satisfying the condition

$$
|f(t, x)| \leq k_{0}+k_{1}|x|, \quad \text { for all }(t, x) \in D,
$$

where $|$.$| is the norm in \mathbb{R}^{n}, k_{0}, k_{1}$ are positive constants. Then there is at least one solution $x(t)$ of the initial value problem (5.1)-(5.2) on the whole interval $[0, a]$.

Proof. Let $U:=\left\{x \in C^{1}\left([0, a], \mathbb{R}^{n}\right):{ }^{C} D_{0}^{\lambda, \alpha, \Psi} x \in C\left([0, a], \mathbb{R}^{n}\right)\right\}$ and let $T: U \rightarrow U$ be the operator defined by the rule

$$
(T x)(t)=e^{-\lambda \Psi(t)} x_{0}+\frac{1}{\Gamma(\alpha)} \int_{0}^{t}[\Psi(t)-\Psi(s)]^{\alpha-1} e^{-\lambda[\Psi(t)-\Psi(s)]} \Psi^{\prime}(s) f(s, x(s)) d s .
$$


The mapping $t \rightarrow(T x)(t)$ is continuous for any $x \in C^{1}\left([0, a], \mathbb{R}^{n}\right)$ and ${ }^{C} D_{a}^{\lambda, \alpha, \Psi}(T x)(t)=f(t, x(t))$ is also continuous on $[0, a]$. This means that the operator $T$ is well-defined, i.e. $T(U) \subset U$. Now, we shall prove that the mapping $T$ satisfies the assumptions of the Schaefer's fixed point theorem.

Claim I: The operator $T$ is continuous.

Let $\left\{x_{n}\right\}_{n=1}^{\infty}, x_{n} \in X$ be a sequence converging to $x \in U$. Then

$$
\begin{aligned}
& \left\|T\left(x_{n}\right)-T(x)\right\|_{C_{a}}=\frac{1}{\Gamma(\alpha)} \max _{t \in[0, a]} \int_{0}^{t}[\Psi(t)-\Psi(s)]^{\alpha-1} e^{-\lambda[\Psi(t)-\Psi(s)]} \Psi^{\prime}(s) \\
& \times\left|f\left(s, x_{n}(s)\right)-f(s, x(s))\right| d s \leq \frac{1}{\Gamma(\alpha)} \int_{0}^{t}[\Psi(t)-\Psi(s)]^{\alpha-1} e^{-\lambda[\Psi(t)-\Psi(s)]} \\
& \quad \times \Psi^{\prime}(s) d s\left(\max _{0 \leq \tau \leq a}\left|f\left(\tau, x_{n}(\tau)\right)-f(\tau, x(\tau))\right|\right) \\
& \leq \frac{1}{\lambda^{\alpha} \Gamma(\alpha)}\left\|f\left(., x_{n}(.)\right)-f(., x(.))\right\|_{C_{a}} \longrightarrow 0 \quad \text { as } n \rightarrow \infty,
\end{aligned}
$$

because $f$ is continuous.

Claim (II): The operator $T$ maps bounded sets into bounded sets in $U$.

Let $x \in B_{r}=\{y \in U:\|y\| \leq r\}$. Then using the condition (5.5) we obtain

$$
\|T(x)\|_{C_{a}} \leq \tilde{r}:=\frac{1}{\lambda^{\alpha}}\left[k_{0}+k_{1} r\right]+\left|x_{0}\right| .
$$

Since the number $\tilde{r}$ is independent of $t$ and $x$, that inequality means the mapping $T$ is uniformly bounded.

Claim (III): $T$ maps bounded sets into equicontinuous sets in $U$.

Let $B_{r}$ as in (II), $x \in B_{r}, t_{1}, t_{2} \in[0, a], t_{1}<t_{2}$ and

$$
K_{\Psi}=\lambda \max _{s \in[0, a]}\left(\Psi^{\prime}(s) e^{-\lambda \Psi(s)}\right) .
$$

Then the mean value theorem implies that $\left|e^{-\lambda \Psi\left(t_{2}\right)}-e^{-\lambda \Psi\left(t_{1}\right)}\right| \leq K_{\Psi}\left|t_{2}-t_{1}\right|$ and we have

$$
\begin{aligned}
& \left|T(x)\left(t_{2}\right)-T(x)\left(t_{1}\right)\right| \leq\left|e^{-\lambda \Psi\left(t_{2}\right)}-e^{-\lambda \Psi\left(t_{1}\right)}\right|\left|x_{0}\right| \\
& \quad+\frac{1}{\Gamma(\alpha)} \mid \int_{0}^{t_{2}}\left[\Psi\left(t_{2}\right)-\Psi(s)\right]^{\alpha-1} e^{-\lambda\left[\Psi\left(t_{2}\right)-\Psi(s)\right]} \Psi^{\prime}(s) f(s, x(s)) d s \\
& \quad-\int_{0}^{t_{1}}\left[\Psi\left(t_{1}\right)-\Psi(s)\right]^{\alpha-1} e^{-\lambda\left[\Psi\left(t_{1}\right)-\Psi(s)\right]} \Psi^{\prime}(s) f(s, x(s)) d s \mid \\
& \leq K_{\Psi}\left|t_{2}-t_{1}\right|+M / \Gamma(\alpha) \\
& \quad \times\left|\int_{0}^{\Psi\left(t_{2}\right)} \sigma^{\alpha-1} e^{-\lambda\left[\Psi\left(t_{2}\right)-\Psi(s)\right]} \Psi^{\prime}(s) d s-\int_{0}^{\Psi\left(t_{1}\right)} \sigma^{\alpha-1} e^{-\lambda\left[\Psi\left(t_{1}\right)-\Psi(s)\right]} \Psi^{\prime}(s) d s\right| \\
& =K_{\Psi}\left|t_{2}-t_{1}\right|+\frac{M}{\Gamma(\alpha)}\left|\int_{0}^{\Psi\left(t_{2}\right)} \sigma^{\alpha-1} e^{-\lambda \sigma} d \sigma-\int_{0}^{\Psi\left(t_{1}\right)} \sigma^{\alpha-1} e^{-\lambda \sigma} d \sigma\right| \\
& =K_{\Psi}\left|t_{2}-t_{1}\right|+\frac{M}{\Gamma(\alpha)}\left|\int_{\Psi\left(t_{1}\right)}^{\Psi\left(t_{2}\right)} \sigma^{\alpha-1} e^{-\lambda \sigma} d \sigma\right| \\
& =K_{\Psi}\left|t_{2}-t_{1}\right|+\frac{M}{\lambda^{\alpha} \Gamma(\alpha)}\left|\int_{\lambda \Psi\left(t_{1}\right)}^{\lambda \Psi\left(t_{2}\right)} \tau^{\alpha-1} e^{-\tau} d \tau\right| .
\end{aligned}
$$


The right hand side of this inequality is independent of $F$ and obviously it converges to 0 as $t_{2} \rightarrow t_{1}$. Therefore, the set $T\left(B_{r}\right)$ is equicontinuous. As a consequence of Claims (I)-(III) with the Arzela-Ascoli theorem, we can conclude that the operator $T$ is completely continuous.

Claim IV: To conclude the proof, we shall show that the set

$$
H:=\{y \in U: y=\mu T(y) \text { for some } \mu \in(0,1)\}
$$

is bounded. Let $x \in H$. Then using the condition (5.5), we have for $t \in[0, a]$,

$$
\begin{aligned}
& |x(t)|=\mu|(T x)(t)| \leq\left|x_{0}\right| \\
& \quad+\frac{1}{\Gamma(\alpha)}\left(\int_{0}^{t}[\Psi(t)-\Psi(s)]^{\alpha-1} e^{-\lambda[\Psi(t)-\Psi(s)]} \Psi^{\prime}(s) d s\right)\left(k_{0}+k_{1}|x(s)|\right) d s \\
& \leq\left|x_{0}\right|+\frac{k_{0}}{\Gamma(\alpha)}\left(\int_{0}^{t}[\Psi(t)-\Psi(s)]^{\alpha-1} e^{-\lambda[\Psi(t)-\Psi(s)]} \Psi^{\prime}(s) d s\right) d s \\
& \quad+\frac{k_{1}}{\Gamma(\alpha)}\left(\int_{0}^{t}[\Psi(t)-\Psi(s)]^{\alpha-1} e^{-\lambda[\Psi(t)-\Psi(s)]} \Psi^{\prime}(s)|x(s)| d s\right) d s \\
& \leq A+\frac{k_{1}}{\Gamma(\alpha)} \int_{0}^{t}[\Psi(t)-\Psi(s)]^{\alpha-1} e^{-\lambda[\Psi(t)-\Psi(s)]} \Psi^{\prime}(s)|x(s)| d s,
\end{aligned}
$$

where $A=\left|x_{0}\right|+k_{0} / \lambda^{\alpha}$. Hence, we have obtained the integral inequality

$$
|x(t)| \leq A+\frac{k_{1}}{\Gamma(\alpha)} \int_{0}^{t}[\Psi(t)-\Psi(s)]^{\alpha-1} e^{-\lambda[\Psi(t)-\Psi(s)]} \Psi^{\prime}(s)|x(s)| d s, \quad t \in[0, a] .
$$

Choose a number $p$ with the property $1<p<\frac{1}{1-\alpha}$. Since $\alpha \in(0,1), \frac{1}{1-\alpha}>1$, such a number $p$ exists and it is clear that $p(\alpha-1)+1>0$. Therefore we can use the Corollary 1. Therefrom and from the Inequality (5.6) it follows that

$$
|x(t)| \leq K=C e^{D \frac{k_{1} \Psi(a)}{\Gamma(\alpha)}}<\infty \forall t \in[0, a],
$$

where

$$
C=2^{\frac{q-1}{q}} A, \quad D=\frac{2^{q-1}}{q} M_{p}^{q}\left(\frac{k_{1}}{\Gamma(\alpha)}\right)^{q} \Psi(a), \quad q=\frac{p}{p-1},
$$

$M_{p}$ is the number from Theorem 2. Hence, we have that

$$
\|x\|_{C_{a}}=\max _{t \in[0, a]}|x(t)| \leq K
$$

and this proves that $H$ is bounded. By the Schaefer's fixed point theorem, the operator $T$ has at least one fixed point.

\section{Application of the Picard's method to tempered $\Psi$-Caputo fractional differential equations}

The method of successive approximations dates to the work of Picard's [34] and it has recently been applied also in the theory of fractional differential 
equations (see e.g. $[22,29,31]$ ). We apply this method in the following second proof of Theorem 3 .

Proof. Let $a>0, B>0$ and

$$
G=\left\{(t, y) \in \mathbb{R} \times \mathbb{R}^{n}: 0 \leq t \leq a,\left|y-e^{-\lambda \Psi(t)} x_{0}\right| \leq B \forall t \in[0, a]\right\} .
$$

Let

$$
M=\max _{(t, x) \in G}|f(t, x)| \text { and } b=\min \{B, \gamma\}, \text { where } \gamma=M / \lambda^{\alpha} .
$$

Define the sequence $\left\{x_{m}\right\}_{m=0}^{\infty}, x_{m} \in C^{0}:=C\left([0, a], \mathbb{R}^{n}\right)$, where

$$
\begin{aligned}
& x_{0}(t)=e^{-\lambda \Psi(t)} x_{0} \forall t \in[0, a], \quad x_{m+1}(t)=e^{-\lambda \Psi(t)} x_{0} \\
& +\frac{1}{\Gamma(\alpha)} \int_{0}^{t}[\Psi(t)-\Psi(s)]^{\alpha-1} e^{-\lambda[\Psi(t)-\Psi(s)]} \Psi^{\prime}(s) f\left(s, x_{m}(s)\right) d s
\end{aligned}
$$

for $m \geq 0, t \in[0, a]$.

First, we need to prove that $\left(t, x_{m}(t)\right) \in G$ for all $m \geq 0$ and for all $t \in[0, a]$. Obviously, $\left(t, x_{0}(t)\right) \in G \forall t \in[0, a]$. Let us estimate

$$
\begin{aligned}
\mid x_{1}(t) & -e^{-\lambda \Psi(t)} x_{0}\left|\leq \frac{1}{\Gamma(\alpha)} \int_{0}^{t}[\Psi(t)-\Psi(s)]^{\alpha-1} e^{-\lambda[\Psi(t)-\Psi(s)]} \Psi^{\prime}(s)\right| f\left(s, x_{0}(s)\right) \mid d s \\
& \leq \frac{M}{\Gamma(\alpha)}\left(\int_{0}^{t}[\Psi(t)-\Psi(s)]^{\alpha-1} e^{-\lambda[\Psi(t)-\Psi(s)]} \Psi^{\prime}(s) d s\right) d s \\
& +\frac{M}{\Gamma(\alpha)}\left(\int_{0}^{\Psi(t)} \sigma^{\alpha-1} e^{-\lambda \sigma}\right) d \sigma \leq \frac{M}{\Gamma(\alpha)} \int_{0}^{\infty} \sigma^{\alpha-1} e^{-\lambda \sigma} d \sigma \\
& =\frac{M}{\Gamma(\alpha)}\left(\frac{\gamma(\alpha)}{\lambda^{\alpha}}\right)=\gamma=\frac{M}{\lambda^{\alpha}} \forall t \in[0, a] .
\end{aligned}
$$

This means that $\left(t, x_{1}(t)\right) \in G \forall t \in[0, a]$. By an easy induction, we have that $\left(t, x_{m}(t)\right) \in G \forall m \geq 1, t \in[0, a]$.

Using the above inequality and the Lipschitz condition we obtain

$$
\begin{aligned}
& \left|x_{2}(t)-x_{1}(t)\right| \leq \frac{L}{\Gamma(\alpha)} \int_{0}^{t}[\Psi(t)-\Psi(s)]^{\alpha-1} e^{-\lambda[\Psi(t)-\Psi(s)]} \Psi^{\prime}(s)\left|x_{1}(s)-x_{0}(s)\right| d s \\
& \quad \leq \frac{M L}{\lambda^{\alpha} \Gamma(\alpha)} \int_{0}^{t}[\Psi(t)-\Psi(s)]^{\alpha-1} e^{-\lambda[\Psi(t)-\Psi(s)]} \Psi^{\prime}(s) d s \\
& \quad=\frac{M L}{\lambda^{\alpha} \Gamma(\alpha)} \int_{0}^{\psi(t)} \sigma^{\alpha-1} e^{-\lambda \sigma} d \sigma \leq \frac{M L}{\lambda^{\alpha} \Gamma(\alpha)} \int_{0}^{\infty} \sigma^{\alpha-1} e^{-\lambda \sigma} d \sigma=\frac{M L}{\left(\lambda^{\alpha}\right)^{2}} \forall t \in[0, a] .
\end{aligned}
$$

This yields the estimate

$$
\left\|x_{2}-x_{1}\right\|_{C_{a}} \leq M L /\left(\lambda^{\alpha}\right)^{2} .
$$

Using this inequality and the Lipschitz condition one can prove the inequality

$$
\left\|x_{3}-x_{2}\right\|_{C_{a}} \leq M L^{2} /\left(\lambda^{\alpha}\right)^{3} .
$$


Hence by the induction, the estimate

$$
\left\|x_{m+1}-x_{m}\right\|_{C_{a}} \leq M L^{m} /\left(\lambda^{\alpha}\right)^{m+1}
$$

holds for all $m \geq 0$. We assume that $\frac{L}{\lambda^{\alpha}}<1$. Therefore, if $m>k$ then

$$
\begin{aligned}
& \left\|x_{m}-x_{k}\right\|_{C_{a}} \leq\left\|x_{m}-x_{k}\right\|_{C_{a}} \leq\left\|x_{m}-x_{m-1}\right\|_{C_{a}}+\left\|x_{m-1}-x_{m-2}\right\|_{C_{a}} \\
& +\cdots+\left\|x_{k+1}-x_{k}\right\|_{C_{a}} \leq \frac{M}{L}\left(\frac{L}{\lambda^{\alpha}}\right)^{m}+\frac{M}{L}\left(\frac{L}{\lambda^{\alpha}}\right)^{m-1}+\cdots+\frac{M}{L}\left(\frac{L}{\lambda^{\alpha}}\right)^{k+1} \\
& \quad \leq \frac{M}{L}\left(\frac{L}{\lambda^{\alpha}}\right)^{k+1} \sum_{i=0}^{\infty}\left(\frac{L}{\lambda^{\alpha}}\right)^{i}=\frac{M}{L}\left(\frac{L}{\lambda^{\alpha}}\right)^{k+1} \frac{1}{1-L / \lambda^{\alpha}} .
\end{aligned}
$$

This yields that

$$
\lim _{k \rightarrow \infty}\left\|x_{m}-x_{k}\right\|_{C_{a}}=0
$$

and that means that $\left\{x_{m}\right\}_{m=0}^{\infty}$ is the Cauchy sequence. Since the space $C_{a}$ is complete, we obtain that there is a mapping $x \in C_{a}$ such that $\lim _{m \rightarrow \infty} x_{m}=x$. The set $G$ is closed and therefore $(t, x(t)) \in G \forall t \in[0, a]$. Therefore we obtain from (6.1) that $x$ is a solution of the initial value problem (5.1)-(5.2). Now we prove that this solution is unique.

Let $u(t), v(t)$ for $t \in[0, a]$ be two different solutions of the initial value problem. Then

$$
\begin{aligned}
& |u(t)-v(t)| \leq \frac{1}{\Gamma(\alpha)} \int_{0}^{t}[\Psi(t)-\Psi(s)]^{\alpha-1} e^{-\lambda[\Psi(t)-\Psi(s)]} \Psi^{\prime}(s)|u(s)-v(s)| d s \\
& \leq \frac{L}{\Gamma(\alpha)} \int_{0}^{t}[\Psi(t)-\Psi(s)]^{\alpha-1} e^{-\lambda[\Psi(t)-\Psi(s)]} \Psi^{\prime}(s) d s\|u-v\|_{C^{0}} \\
& \leq \frac{L}{\Gamma(\alpha)} \int_{0}^{\infty} \sigma^{\alpha-1} e^{-\lambda \sigma} d \sigma\|u-v\|_{C^{0}}=\frac{L}{\lambda^{\alpha}}\|u-v\|_{C_{a}} .
\end{aligned}
$$

This yields the inequality

$$
\|u-v\|_{C_{a}} \leq \frac{L}{\lambda^{\alpha}}\|u-v\|_{C_{a}} .
$$

Since $\gamma=\frac{L}{\lambda^{\alpha}}<1$, the inequality is valid if and only if $u(t)=v(t) \forall t \in[0, a]$. The constant $\gamma$ is independent of $a$ and therefore the solution $x(t)$ is global.

\section{Representation of solutions of linear systems of $\Psi$-Caputo fractional differential equations}

Consider the initial value problem

$$
\begin{aligned}
{ }^{C} D_{0}^{\alpha, \lambda, \Psi} x(t) & =A x(t), \quad x \in \mathbb{R}^{n}, \alpha \in(0,1), \\
x(0) & =x_{0} \in \mathbb{R}^{n},
\end{aligned}
$$


where $A$ is a constant matrix, $\lambda>0$. Consider also the initial value

$$
\begin{aligned}
{ }^{C} D_{0}^{\alpha, \Psi} y(t) & =A x(t), \quad y \in \mathbb{R}^{n}, \alpha \in(0,1), \\
y(0) & =x_{0} \in \mathbb{R}^{n} .
\end{aligned}
$$

We apply the Picard's successive approximations to prove the following theorem.

Theorem 6. The following assertions hold:

1. The solution of the initial-value problem (7.3)-(7.4) is given by the formula

$$
y(t)=E_{\alpha}\left(A[\Psi(t)-\Psi(0)]^{\alpha}\right) x_{0},
$$

where $E_{\alpha}(A)=E_{\alpha, 1}(A)$,

$$
E_{\alpha, \beta}(A)=\sum_{i=0}^{\infty} \frac{A^{i}}{\Gamma(i \alpha+\beta)}, \alpha>0, \beta>0
$$

is the Mittag-Leffler matrix-valued function with two parameters.

2. The solution $x(t)$ of the initial-value problem (7.1)-(7.2) is given by the formula

$$
x(t)=e^{-\lambda \Psi(t)} E_{\alpha}\left(A[\Psi(t)-\Psi(0)]^{\alpha}\right) x_{0} .
$$

Proof. Let us prove the first assertion. From Definition 4 and the second assertion of Lemma 1 it follows that the initial-value problem (7.3)-(7.4) is equivalent to the integral equation

$$
y(t)=x_{0}+\frac{1}{\Gamma(\alpha)} \int_{0}^{t}[\Psi(t)-\Psi(s)]^{\alpha-1} \Psi^{\prime}(s) A y(s) d s .
$$

We apply the Picard's successive approximations to find a solution of the Equation (7.7). One can show by the method of induction (see also the proof of $[1$, Theorem 2]) that these approximations are defined as follows:

$$
y_{0}(t)=x_{0}, \quad y_{m}(t)=\sum_{i=0}^{m} \frac{A^{i}[\Psi(t)-\Psi(0)]^{i \alpha}}{\Gamma(i \alpha+1)} x_{0}, m \geq 1 .
$$

From the second proof of Theorem 3 it follows that the sequence $\left\{y_{m}(t)\right\}_{m=0}^{\infty}$ is uniformly convergent on an arbitrary compact interval $[0, a], a>0$ to a continuous function $y(t)$, which is the unique solution of the Equation (7.7). Hence, the formula for the solution of the initial-value problem (7.3)- (7.4) has the form

$$
y(t)=\sum_{i=0}^{\infty} \frac{A^{i}[\Psi(t)-\Psi(0)]^{i \alpha}}{\Gamma(i \alpha+1)} x_{0}=E_{\alpha}\left(A[\Psi(t)-\Psi(0)]^{\alpha}\right) x_{0} .
$$

Now, let us prove the second assertion. Let $x(t)$ be a solution of the problem (7.1)-(7.2). From the equality (3.1) it follows that

$$
{ }^{C} D_{0}^{\alpha, \lambda, \Psi} x(t)=e^{-\lambda \Psi(t) C} D_{0}^{\alpha, \Psi}\left(e^{\lambda \Psi(t)} x(t)\right)=A x(t)
$$


and hence

$$
{ }^{C} D_{0}^{\alpha, \Psi}\left(e^{\lambda \Psi(t)} x(t)\right)=A\left(e^{\lambda \Psi(t)} x(t)\right) .
$$

This means that $y(t)=e^{\lambda \Psi(t)} x(t)$ is a solution of the problem (7.3)-(7.4). From the first assertion it follows that $y(t)$ has the form (7.5) and hence $x(t)$ is given by the formula (7.6).

\section{Example}

Consider the following initial-valued problem for the tempered $\Psi$-Caputo fractional differential equation with $\Psi(t)=\omega t, \omega>0$ :

$$
\begin{aligned}
{ }^{C} D_{0}^{\alpha, \lambda, \omega t} x(t) & =A x(t), \quad t>0, \alpha \in(0,1), \\
x(0) & =x_{0} \in \mathbb{R}^{n},
\end{aligned}
$$

where $A$ is a constant matrix, $\lambda>0$. By the Theorem 6 the solution $x(t)$ of the problem (8.1)-(8.2) has the form

$$
x(t)=e^{-(\lambda \omega) t} E_{\alpha}\left(A\left[\omega^{\alpha} t^{\alpha}\right]\right) x_{0}=e^{-\lambda \omega t} E_{\alpha}\left(\left(\omega^{\alpha} A\right)\left[t^{\alpha}\right]\right) x_{0} .
$$

We have obtained that $x(t)$ is a solution of the $(\lambda \omega)$-tempered Caputo fractional initial value problem

$$
\begin{aligned}
{ }^{C} D_{0}^{\alpha, \lambda \omega} x(t) & =\left(\omega^{\alpha} A\right) x(t), \quad t>0, \alpha \in(0,1), \\
x(0) & =x_{0} \in \mathbb{R}^{n},
\end{aligned}
$$

defined in the paper [23] (see also [12] and [32]).

\section{Conclusions and a future work}

In the present paper, we proposed a new type of fractional derivative, which we call the tempered $\Psi$-Caputo fractional derivative. We obtained some existence and uniqueness results for differential equations involving this fractional derivative. The proof of the existence theorem is based on an application of the Schaefer's fixed point theorem. In the proof of the existence and uniqueness theorem Picard's successive approximation method was used. The uniqueness of solutions is proved by applying a new integral inequality of the Henry-Gronwall type for integral inequality with tempered $\Psi$-fractional integral. Its proof is based on a desingularization method, introduced by the first author in the papers [26] and [27]. Applying this method we obtained an exponential estimate, very useful for applications, mainly in the stability theory for this new type of fractional differential equations. In our opinion, there are many possibilities for applications of the new fractional derivative. The estimates derived in the proofs show that this derivative has very good properties, suitable for the study of asymptotic properties of solutions of the new fractional differential equations, including their stability.

Problems concerning fractional differential equations with $\Psi$-Caputo and $\Psi$-Hilfer derivatives, studied in the papers mentioned in introduction, can 
be studied also with tempered $\Psi$-Caputo and $\Psi$-Hilfer derivatives, respectively. The aim of our future work is to study stability properties of tempered $\Psi$-fractional differential equations with delays. In this context, the Katugampola-type fractional differential equations could be interesting (see [18] and $[17])$.

\section{Acknowledgements}

The authors would like to express their gratitude to the anonymous referees for their valuable comments which significantly improved the original manuscript. The work of the first author was supported by the Slovak Research and Development Agency under the contract No. APVV-18-0308 and by the Slovak Grant Agency VEGA-SAV-MŠ No. 1/0358/20.

\section{References}

[1] T. Abdeljawad, F. Madjidi, F. Jarad and N. Sene. On dynamic systems in the frame of singular function dependent kernel fractional derivatives. Mathematics, 7(10):946-959, 2019. https://doi.org/10.3390/math7100946.

[2] M.S. Abdo, A.G. Ibrahim and S.K. Panchal. Nonlinear implicit fractional differential equation involving $\psi$-Caputo fractional derivative. Proceedings of the Jangjeon Mathematical Society, 22(3):387-400, 2019.

[3] M.S. Abdo, A.G. Ibrahim and H.A. Wahash. Ulam-Hyers-Mittag-Leffler stability for $\psi$-Hilfer problem with fractional order and infinite delay. Results in Appl. Math., 7:100115, 2020. https://doi.org/10.1016/j.rinam.2020.100115.

[4] M.S. Abdo, K.Shah, S.K. Panchal and H.A. Wahash. Existence and Ulam stability results of a coupled system for terminal value problems involving $\psi$-Hilfer fractional operator. Adv. Differ. Equ., 2020(316), 2020. https://doi.org/10.1186/s13662-020-02775-x.

[5] M.S. Abdo, S.K. Panchal and H.S. Hussein. Fractional integro-differential equations with nonlocal conditions and $\psi$-Hilfer fractional derivative. Proc. Indian Acad. Sci., 24(4):564-584, 2019. https://doi.org/10.3846/mma.2019.034.

[6] M.S. Abdo, S.K. Panchal and A.K. Saeed. Fractional boundary value problem with $\psi$-Caputo fractional derivative. Proc. Indian Acad. Sci., 129(65):64-78, 2019. https://doi.org/10.1007/s12044-019-0514-8.

[7] M.S. Abdo, S.T. Thabet and B. Ahmad. The existence and Ulam-Hyers stability results for $\psi$-Hilfer integrodifferential equations. J. Pseudo-Differ. Oper. Appl., 11(131):1757-1780, 2020. https://doi.org/10.1007/s11868-020-00355-x.

[8] R. Almeida. A Caputo fractional derivative of a function with respect to another function. Commun. in Nonlinear Sci. and Numer. Simulation, 44:460-481, 2017. https://doi.org/10.1016/j.cnsns.2016.09.006.

[9] R. Almeida. A Gronwall inequality for general Caputo fractional operator. Math. Ineq. and Appl., 20(4):1089-1105, 2017. https://doi.org/10.7153/mia-2017-2070.

[10] R. Almeida, A.B. Malinowska and M.T.T. Monteiro. Fractional differential equations with a Caputo derivative with respect to a kernel function and their applications. Math. Meth. Appl. Sci., 41:336-352, 2018. https://doi.org/10.1002/mma.4617. 
[11] G. Butler and T. Rogers. A generalization of a lemma of Bihari and applications to piecewise estimates for integral equations. J. Math. Anal. Appl., 33(1):77-81, 1971. https://doi.org/10.1016/0022-247X(71)90183-1.

[12] M. Chen and W. Deng. Discrete fractional substantial calculus. ESAIM: Math. Modell. Numer., 49(2):373-394, 2015. https://doi.org/10.1051/m2an/2014037.

[13] M. Fečkan, J.R. Wang and M. Pospíšil. Fractional-Order Equations and Inclusions. De Gruyter, Berlin, Boston, 2017. https://doi.org/10.1515/9783110522075.

[14] S. Harikrishnan, K. Kanagarajan and D. Vivek. Existence and stability results for boundary value problem for differential equation with $\psi$-Hilfer fractional derivative. J. Appl. Nonlinear Dynamics, 8(2):251-259, 2019. https://doi.org/10.5890/JAND.2019.06.008.

[15] D. Henry. Geometric Theory of Semilinear Parabolic Equations. Springer-Verlag, Berlin, Heidelberg, New York, 1981. https://doi.org/10.1007/BFb0089647.

[16] R. Hilfer. Applications of Fractional Calculus in Physics. World Scientific, Singapore, 2000. https://doi.org/10.1142/9789812817747.

[17] M. Jamaki, K. Kanagarajan and E.M. Elsayed. Katugampola-type fractional differential equations with delay and impulses. Mathematical and Theoretical Physics, 1(3):73-77, 2018. https://doi.org/10.15406/oajmtp.2018.01.00012.

[18] U.N. Katugampola. New approach to a generalized fractional integral. Appl. Math. Comput., 218(3):860-865, 2011. https://doi.org/10.1016/j.amc.2011.03.062.

[19] A. Kilbas, H.M. Srivastava and J.J. Trujillo. Theory and Applications of Fractional Differential Equations. North-Holland Mathematics Studies 204. Amsterdam, Boston, Heidelberg, London, 2006.

[20] K.D. Kucche and J.P. Kharade. Analysis of impulsive $\phi$-Hilfer fractional differential equations. Mediterr. J. Math., 17(5), 2020. https://doi.org/10.1007/s00009-020-01575-7.

[21] K.D. Kucche, A.D. Mali and V.C. Da Sousa. On the nonlinear $\psi$-Hilfer fractional differential equations. Computational and Appl.Math., 38(2), 2019. https://doi.org/10.1007/s40314-019-0833-5.

[22] V. Lakshmikantham, L. Leela and J. Vasundra Devi. Theory of Fractional Dynamic Systems. Cambridge Scientific Publ., Cambridge, 2009.

[23] C. Li, W. Deng and L. Zhao. Well-posedness and numerical algorithm for the tempered fractional ordinary differential equations. Discrete and Continuous Dynamical Systems, Series B, 24(4):1989-2015, 2019. https://doi.org/10.3934/dcdsb.2019026.

[24] J. Liouville. Memoire sur quelques question de geometrie et de mecanique et sur un nouveau genre de calcul pour resudre ces question. J. Ecole Polytech., 13(21):1-69, 1832.

[25] K. Liu, J.R. Wang and D. O'Regan. Ulam-Hyers-Mittag-Leffler stability for $\psi$-Hilfer fractional-order delay differential equations. Adv. Differ. Equ., 50:112, 2019. https://doi.org/10.1186/s13662-019-1997-4.

[26] M. Medved. A new approach to an analysis of Henry type integral inequalities and their Bihari type versions. J. Math. Anal. Appl., 214(2):349-366, 1997. https://doi.org/10.1006/jmaa.1997.5532. 
[27] M. Medved. Integral inequalities and global solutions of semilinear evolution equations. J. Math. Anal. Appl., 267(2):634-650, 2002. https://doi.org/10.1006/jmaa.2001.7798.

[28] M. Medved and E. Brestovanská. Corrigendum to the paper: New conditions for the exponential stability of fractionally perturbed ODEs. Electron. J. Qual. Theory Differ. Equ., 102:1-2, 2018. https://doi.org/10.14232/ejqtde.2018.1.102.

[29] M. Medved and E. Brestovanská. New conditions for the exponential stability of fractionally perturbed ODEs. Electron. J. Qual. Theory Differ. Equ., 84:1-14, 2018. https://doi.org/10.14232/ejqtde.2018.1.84.

[30] M. Medved and E. Brestovanská. Sufficient conditions for the exponential stability of nonlinear fractionally perturbed ODEs with multiple delays. Fract. Differ. Calc., 9(2):263-278, 2019. https://doi.org/10.7153/fdc-2019-09-17.

[31] M. Medved and M. Pospíšil. On the existence and exponential stability for differential equations with multiple constant delays and nonlinearity depending on fractional substantial integrals. Electron. J. Qual. Theory Differ. Equ., 43:117, 2019. https://doi.org/10.14232/ejqtde.2019.1.43.

[32] M.L. Morgado and M. Rebelo. Well-posedness and numerical approximation of tempered fractional terminal value problems. Fract. Calc. Appl. Anal., 20(5):1239-1262, 2017. https://doi.org/10.1515/fca-2017-0065.

[33] I. Petráš. Fractional-Order Nonlinear Systems, Modeling, Analysis and Simulation. Springer, Heidelberg, Dortrecht, London, New York, 2011. https://doi.org/10.1007/978-3-642-18101-6.

[34] E. Picard. Mémoire sur la theórie des equations aux dérivés partielles et la méthode des approximations successives. J. de Math. Pures Appl., 231(6):145210, 1890.

[35] I. Podlubný. Fractional Differential Equations. Academic Press, San Diego, 1999.

[36] B. Riemann. Versuch einer allgemainen auffasung der integration und differentiation. Gesammelte Werke, 1876.

[37] F. Sabzibar, M.M. Meerschaert and J. Chen. Tempered fractional calculus. J. Comput. Physics, 293:14-28, 2015. https://doi.org/10.1016/j.jcp.2014.04.024.

[38] H. Schaefer. Über die Methode der a Priori-Schranken. Math. Ann., 129:415416, 1955. https://doi.org/10.1007/BF01362380.

[39] J.C. Sousa and E.C. De Oliveira. On the $\psi$-Hilfer fractional derivative. Commun. Nonlinear Sci. Numer. Simul., 60:72-91, 2018. https://doi.org/10.1016/j.cnsns.2018.01.005.

[40] J.C. Sousa and E.C. De Oliveira. On the Ulam-Hyers-Rassias stability of nonlinear differential equations using the $\psi$-Hilfer operator. J. Fixed Point Theory and Appl., 20(3):1-21, 2018. https://doi.org/10.1007/s11784-018-0587-5.

[41] J.C. Sousa and E.C. De Oliveira. A Gronwall inequality and the Cauchy-type problems by means of $\psi$-Hilfer operator. Differ. Equ. Appl., 11(1):87-106, 2019. https://doi.org/10.7153/dea-2019-11-02.

[42] J.C. Sousa and E.C. De Oliveira. On the $\psi$ - fractional integral and applications. Comp. Appl. Math., 38(4), 2019. https://doi.org/10.1007/s40314-019-0774-z.

[43] J.C. Sousa, K.D. Kucche and E.C. De Oliveira. Stability of $\psi$-Hilfer impulsive fractional equations. Applied Mathematics Letters, 88:73-80, 2019. https://doi.org/10.1016/j.aml.2018.08.013. 
[44] J.C. Sousa, F.G. Rodrigues and E.C. De Oliveira. Stability of the Volterra integro-differential equations by means of $\psi$-Hilfer operator. Mathematical Methods in the Applied Sciences, 12, 2019. https://doi.org/10.1002/mma.5563.

[45] V.E. Tarasov. Fractional Dynamics: Application of Fractional Calculus to Dynamics of Particles. Fields and Media. Springer, New York, 2010.

[46] D. Vivek, E.M. Elsayed and K. Kanagarajan. Theory and analysis of $\psi$-fractional differential equations with boundary conditions. Commun. Appl. Anal., 22(3):401-414, 2018.

[47] H. Ye, J. Gao and Y. Ding. A generalized Gronwall inequality and its application to a fractional differential equations. J. Math. Anal. Appl., 328(2):1075-1081, 2007. https://doi.org/10.1016/j.jmaa.2006.05.061.

[48] Y. Zhou. Basic Theory of Fractional Differential Equations. World Scientific, New Jersey, London, Singapore, 2014. https://doi.org/10.1142/9069. 